\title{
A Novel Hydrate Form of Sodium Dodecyl Sulfate and Its Crystallization Process
}

\author{
Hung Lin Lee, Yun Sheng Cheng, Kuan Lin Yeh, Tu Lee* \\ Department of Chemical and Materials Engineering, National Central University, \\ 300 Zhongda Road, Zhongli District, Taoyuan City 32001, Taiwan R.O.C.
}

\section{Corresponding Author}

Professor Tu Lee. E-mail: tulee@cc.ncu.edu.tw

\section{This PDF file includes:}

Form spaces of sodium dodecyl sulfate (SDS) and sodium sulfate (SS) in Tables S1 and S2, TGA scans and FTIR spectra of purchased SDS, SDS $1 / 8$ hydrate and the novel SDS hydrate in Figures S1 and S2, DLS scans of 20wt\% SDS (aq)-acetone solutions in Figure S3, and FTIR, Raman and PXRD of SS in Figures S4 to S6. 


\section{TABLES}

Table S1. Form Space of purchased SDS.

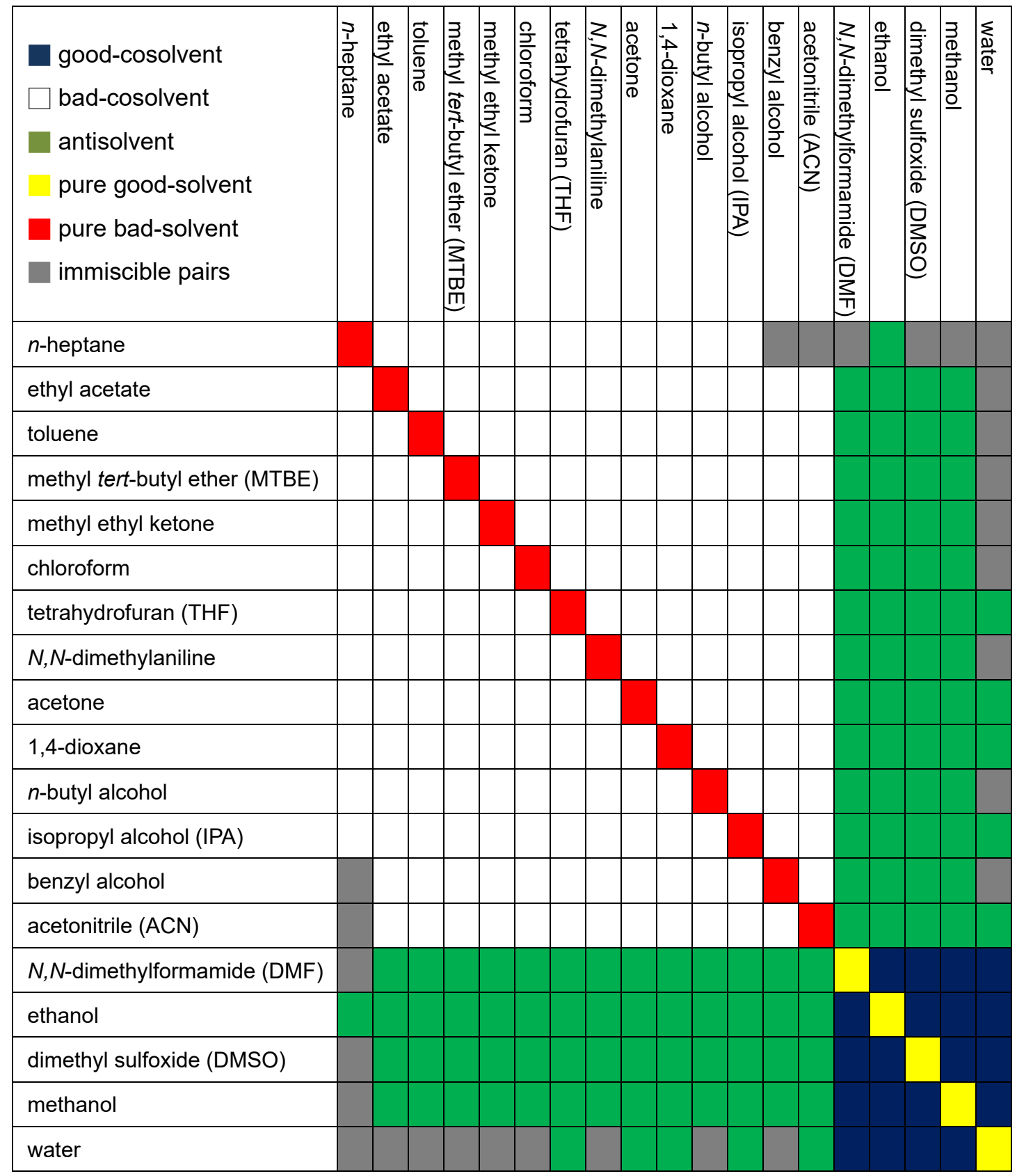


Table S2. Form Space of SS.

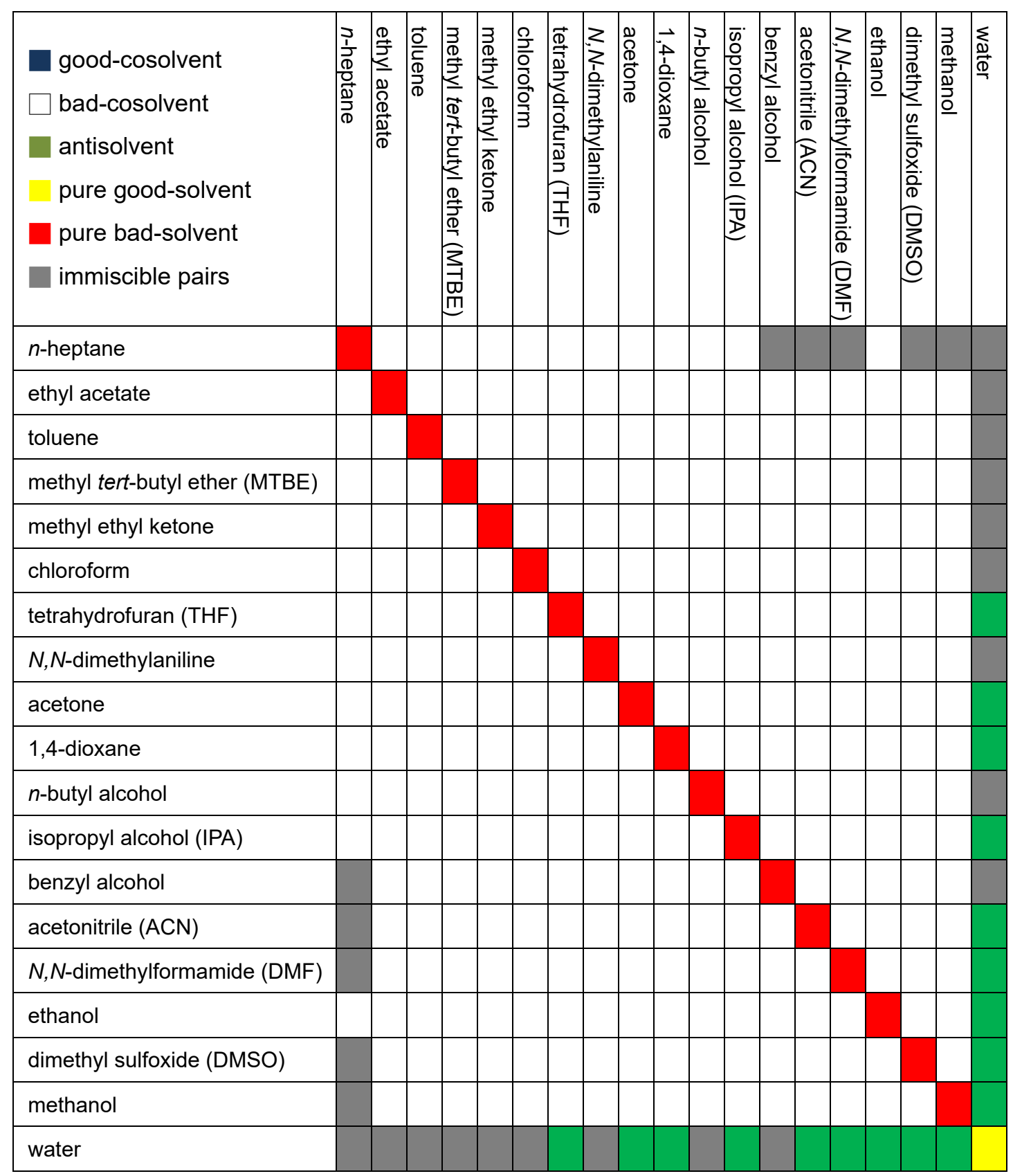




\section{FIGURES}

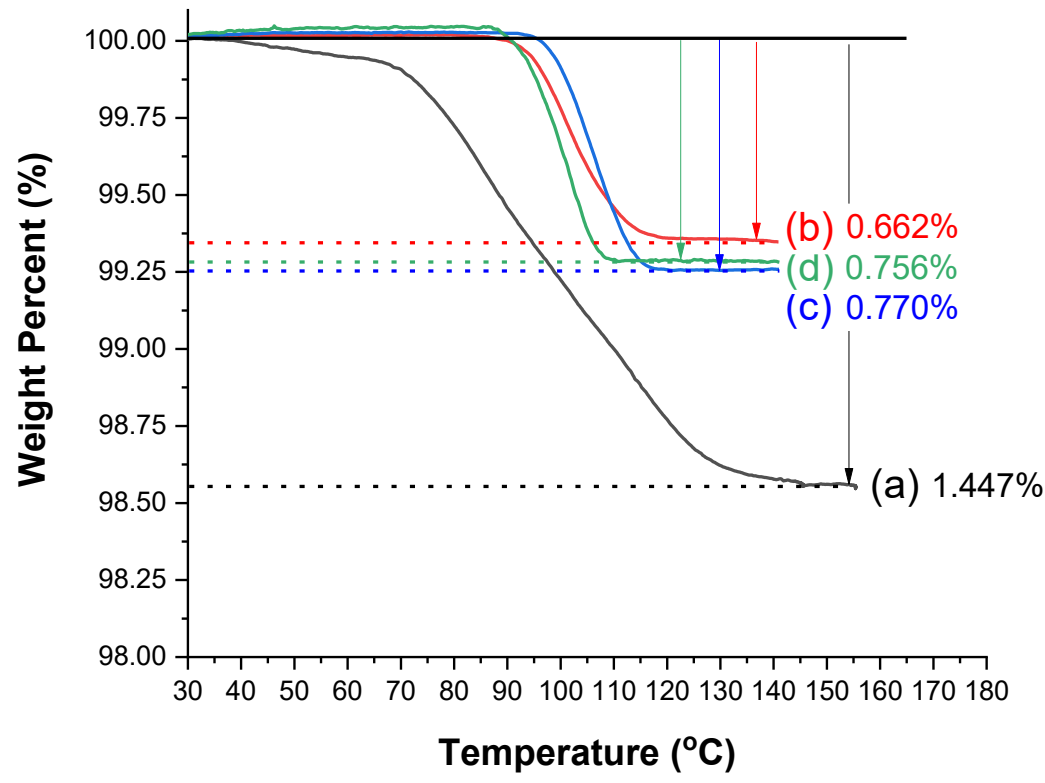

Figure S1. TGA scans of one purchased SDS (Lot. MKBX0092V) (a) upon 1st heating to $155^{\circ} \mathrm{C}$ and (b) upon 2 nd heating after dehydration, and the ones of our prepared SDS $\cdot 1 / 8$ hydrate (c) upon 1st heating to $140^{\circ} \mathrm{C}$ and (d) upon 2 nd heating after dehydration. The dehydration was completed by 1 st heating to the two temperatures (i.e. $155^{\circ}$ and $140^{\circ} \mathrm{C}$ ) until their weights remained unchanged without any loss due to the removal of hydrate(s). 


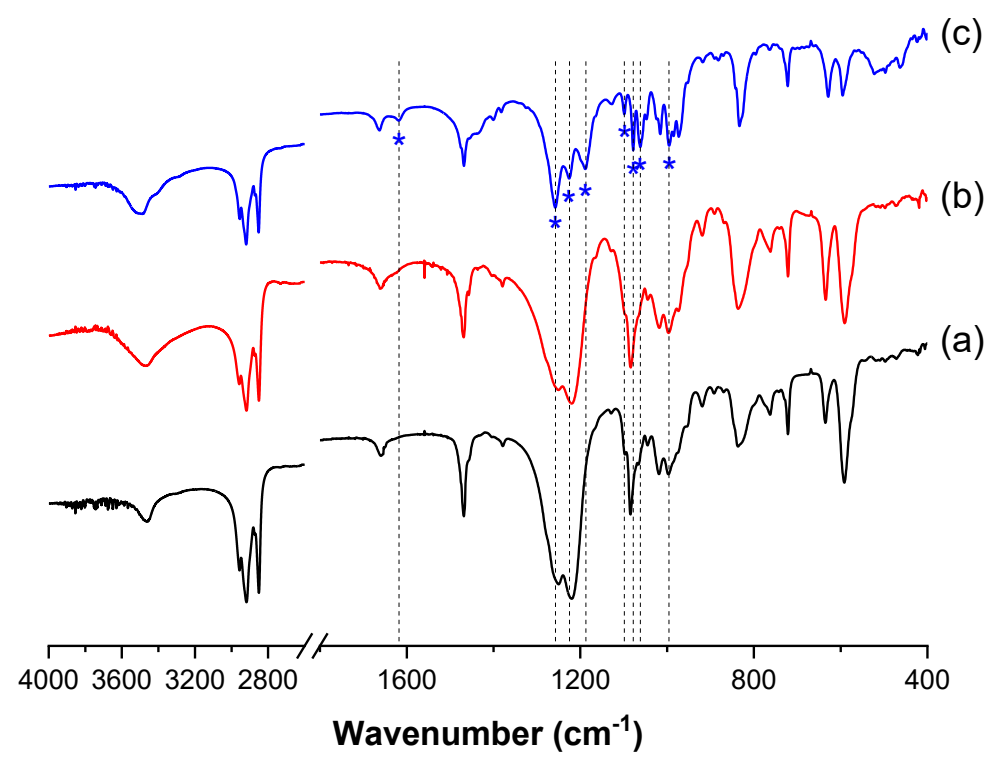

Figure S2. FTIR spectra of (a) purchased SDS, (b) SDS $1 / 8$ hydrate, and (c) the novel SDS hydrate (the asterisks denoted the characteristic peaks at 1618, 1257, 1225, 1188, 1099, 1078, 1061 , and $996 \mathrm{~cm}^{-1}$ for the novel SDS hydrate). 


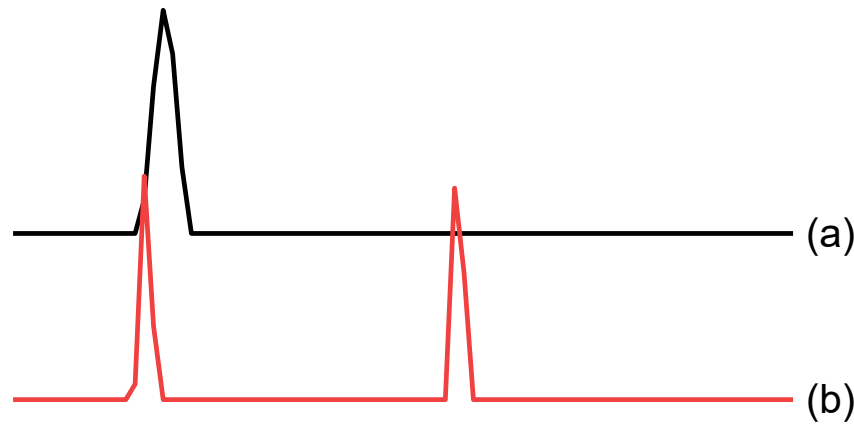

(b)

(c)

(d)

(e)

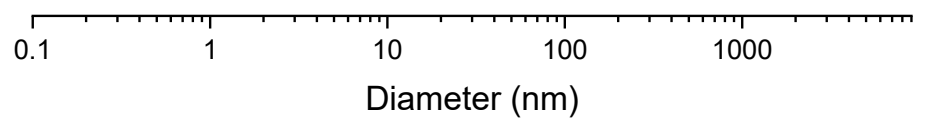

Figure S3. DLS scans of (a) $8.2 \mathrm{mM}$ SDS (aq), which is the critical micelle concentration, (b) $20 \mathrm{wt} \%$ SDS (aq), and the solutions consisting of $20 \mathrm{wt} \%$ SDS (aq) and acetone at ratios of (c) 1:1 (v/v), (d) 1:2(v/v) and (e) 1:3(v/v). 


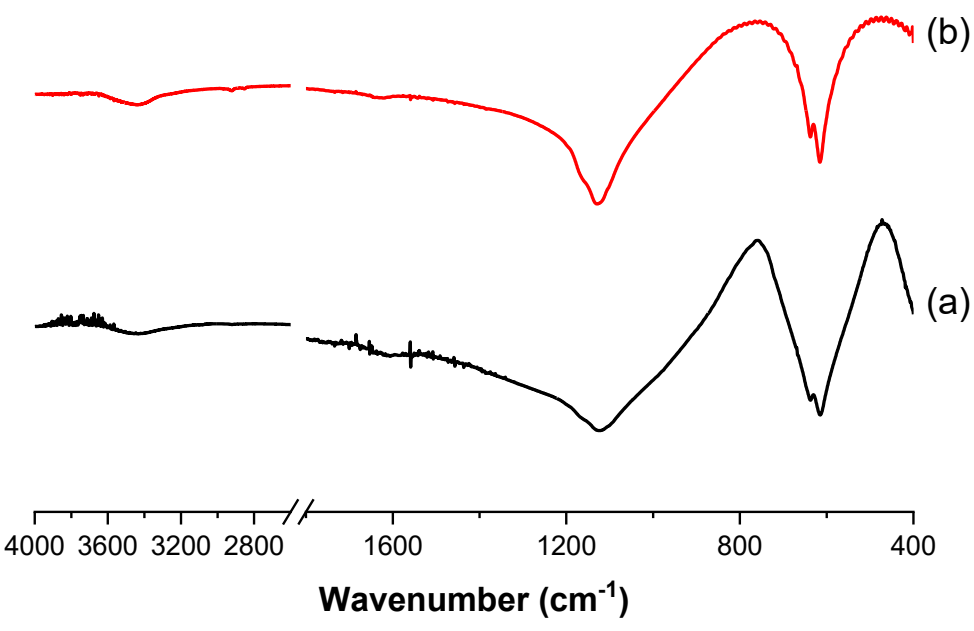

Figure S4. FTIR spectra of (a) purchased SS, and (b) SS produced upon the addition of acetone after synthesis of SDS. 


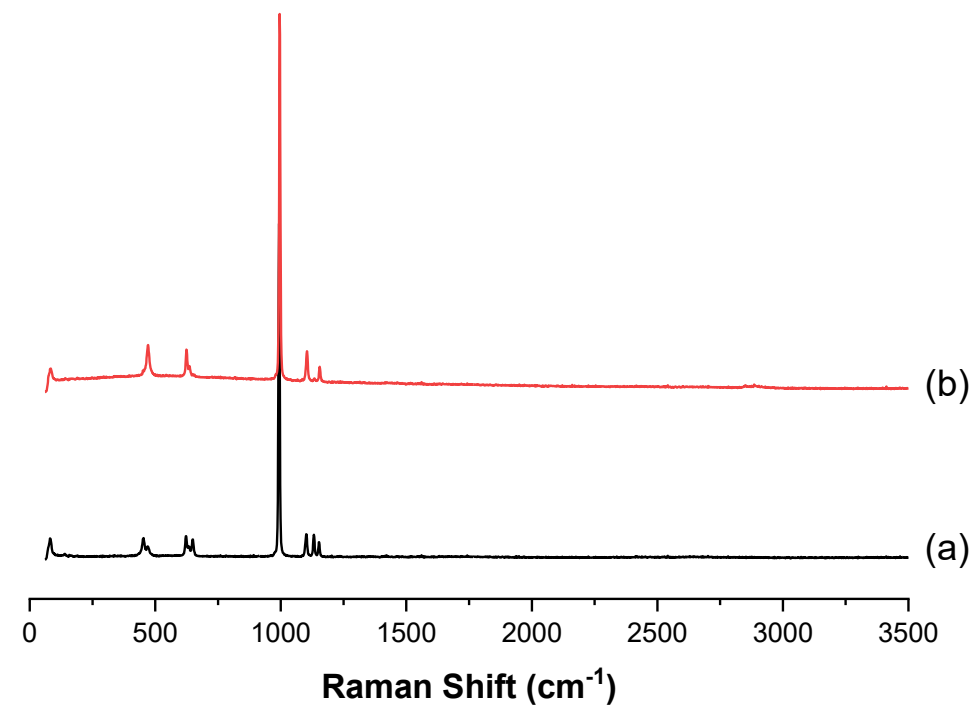

Figure S5. Raman spectra of (a) purchased SS, and (b) SS produced upon the addition of acetone after synthesis of SDS. 


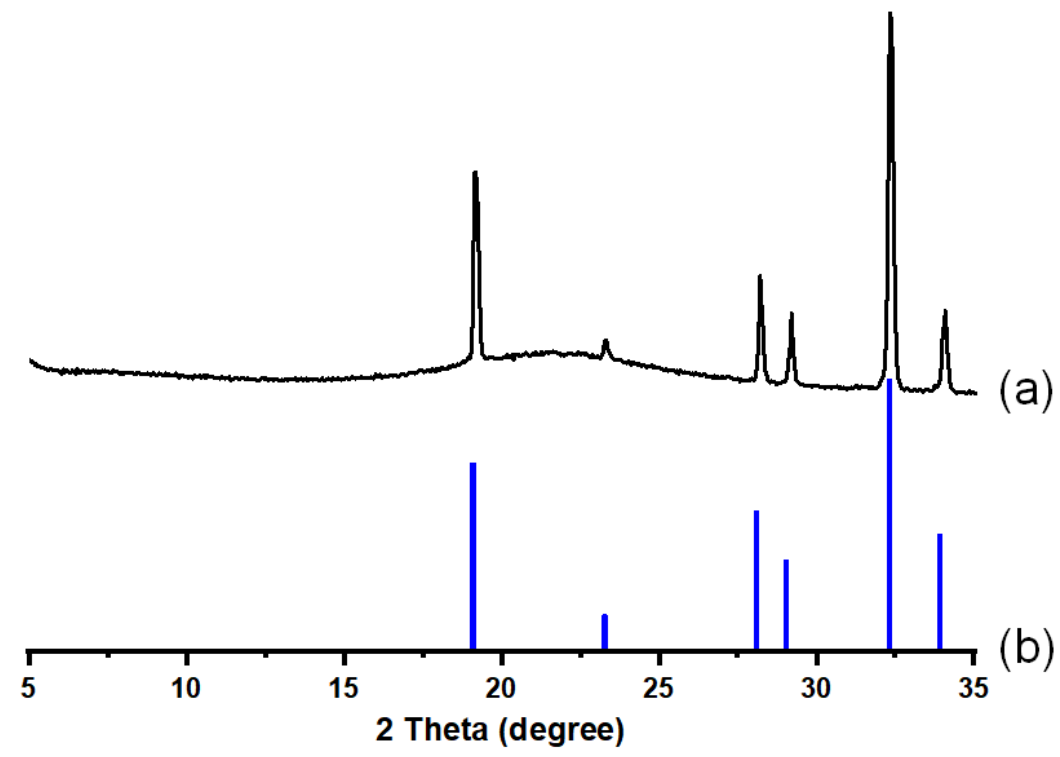

Figure S6. (a) PXRD patterns of SS produced upon the addition of acetone after synthesis of SDS, and (b) theoretical pattern of anhydrous SS from the Crystallography Open Database (COD) with COD ID: 1011184. 